\title{
Personality across Diverse Sexual Orientations and Gender Identities in an Online Convenience Sample
}

\begin{abstract}
Sexual and gender minority (SGM) participants are generally underrepresented in quantitative psychology research. This may affect the generalizability of past research and overlooks a growing portion of the world's population. Previous surveys report differences in the Big Five between limited categorical designations of gender and sexual orientation (e.g., excluding pansexual or nonbinary people) and estimated at the level of factors, rather than finergrained facets. The current study sought to characterize the feasibility of recruiting an SGMdiverse sample online by surveying personality and sexuality measures on the Reddit social media platform and to test the gender similarities hypothesis. We report group differences using three approaches (dichotomous, categorical, and dimensional) at the level of personality factors and facets. In preregistered analyses of 1,259 participants (73\% identifying as SGM), we found that data quality was high, personality differences between groups were small overall $\left(|d|_{\text {average }}=\right.$ $\left.0.16 ;|d|_{\text {range }}=0-0.66\right)$, and there was substantial variability between facet-level group differences $(|d| \pm 0.59)$. We discuss implications for the attainability of diverse samples, group comparisons, and representativeness.
\end{abstract}

Keywords: internet research; sexual and gender minority; recruitment; measurement invariance; personality 


\section{Personality Across Diverse Sexual Orientations and Gender Identities in an Online Convenience Sample}

Psychological research as a whole tends to focus on socially dominant groups to the exclusion of participants with marginalized identities (e.g., Buchanan et al., 2021; Gosling et al., 2010; Henrich et al., 2010). In explorations of comparisons between gender identities or sexual orientations, research is often limited to binary distinctions, such as gay versus straight and men versus women. Sexual orientation and gender identities are much more complex than binary, or even categorical, distinctions, yet we typically do not have sufficient representation of these diverse subgroups (National Academies of Sciences, Engineering, and Medicine, 2020). Furthermore, when groups are compared, assumptions are made that any observed differences are due to the identity group membership itself, rather than social or structural factors that cooccur with marginalized identities. (Although the current study addresses diversity among sexual and gender identities, the same principles apply to other marginalized identities, like Black, Indigenous, and people of color; Buchanan et al., 2021.) The current study sought to characterize the general pattern (or lack) of differences in personality factors and facets across diverse gender identities and sexual orientations, examined across a variety of methods of classifying identities.

Misclassifying or ignoring gender and sexual orientation has far-reaching implications for health institutions, public policy, and science. While sexual and/or gender minority (SGM) people have been identified as a health disparity population for research (Pérez-Stable, 2016), subgroups within the SGM umbrella have different experiences and needs (health of gender versus sexual minority (Haas et al., 2010; Mustanski et al., 2010); health of gay/lesbian versus bisexual (Cochran \& Mays, 2009; Feinstein \& Dyar, 2017; Jorm et al., 2002); intersectional transgender sexual minorities (Miguel \& Tobias, 2019)). It is challenging to estimate the overall 
prevalence of SGM identity in the general population, due to both highly variable response options and ongoing social (and legal) stigmatization around the world which impacts willingness to report. However, SGM identities are not rare. Overall, SGM identities might encompass 12-14\% of the U.S. adult population (Anderson et al., 2021; Jones, 2021; Wilson \& Meyer, 2021). These estimates amount to substantial expected and potential participation across the huge number of psychological studies that are conducted. Common arguments for the exclusion of groups outside of the traditional gender binary often rest on the difficulty in recruiting such minority participants; however, these arguments are problematic in nature (Cameron \& Stinson, 2019). Treating gender as a static binary misaligns social scientists’ understanding of gender processes and undermines growing social acceptance and support for transgender and nonbinary people (Cameron \& Stinson, 2019). The present research sought to address the feasibility of gender- and sexuality-inclusive research by examining the validity of using an online convenience sampling approach, namely, responses to an unpaid survey posted on the social media platform Reddit.

\section{Gender and Sexual Orientation Differences in Personality}

The Big Five - extraversion, agreeableness, conscientiousness, neuroticism, and openmindedness - represent broad patterns of thinking, feeling, and behaving that are expected to differ from person to person (John et al., 2008). Any differences that manifest between groups may be evidence of the context—including social structures, age, or sociopolitical climate—in which the measurement occurs (Harden \& Koellinger, 2020; Hyde, 2005). For example, an analysis of over 17,637 participants from 55 countries concluded that there were widespread apparent group differences between men and women in nations with high gender egalitarianism on all but one of the Big Five (conscientiousness, extraversion, agreeableness, and neuroticism). 
These gender differences were more pronounced within nations in North America, Oceania, and parts of Europe than in other worldwide locations (Schmitt et al., 2008). Early meta-analyses spanning 1940 to 1992 reported higher anxiety in women than men, as well as extraversion, trust, and nurturance, whereas men were more assertive (Feingold, 1994). Similar results were found again when summarizing differences between women and men on the NEO Personality Inventory (Costa et al., 2001). One of the least understood personality factors, open-mindedness, may be related to identity formation and so is proposed to be especially beneficial for lesbian/gay and bisexual populations (Zoeterman \& Wright, 2014). Although personality is often discussed at the level of broad factors (e.g., the Big Five), such constructs may be more narrowly broken down into facet-level information. Personality factors serve for parsimony, but each factor covers a relatively large content space and when we average scores to these higher levels, we sacrifice specificity. It is at the narrower construct level of facets that patterns of differences might be more likely to emerge (Mõttus et al., 2020).

Previous research has suggested average differences in the Big Five personality factors between men and women, as well as between bisexual, heterosexual, and homosexual people. A recent meta-analysis drew from studies published from 1972 to 2020, in which samples were clinical populations, non-U.S. national probability samples, and a few convenience samples. Although the findings suggested the presence of sporadic small to moderate group differences $(|d|=0-0.45 ;$ Allen \& Robson, 2020), there are notable gaps in the available data which limit the ability to draw conclusions about differences between specific group identities versus differences appearing simply between dominant and marginalized identities. Specifically, nonbinary, genderqueer, and/or gender non-conforming people were not included, nor were pansexual or asexual participants. One study on the HEXACO model of personality included asexual 
respondents but was similarly limited only to men and women (Bogaert et al., 2018). These reports, and similar comparative approaches across other domains (subjective well-being: BatzBarbarich et al., 2018; loneliness: Maes et al., 2019; self-esteem: Kling et al., 1999; depression and anxiety: Ross et al., 2018), continue to exclude a variety of sexual orientation and gender identities that are increasing in prevalence in the cultures that are most often studied in psychology.

\section{The Similarities Hypothesis}

We approach the current study of the relationship between personality and diverse SGM identities from the perspective that largely no gender or sexual orientation differences exist systematically on psychological variables, also known as the gender similarities hypothesis (Hyde, 1990, 2005). The gender similarities hypothesis states that on most, but not all, psychological variables males and females do not differ (Hyde, 2005). It goes on to propose two more hypotheses about differences. First, any group differences are expected not to be large systematically. Evidence from meta-analyses and meta-synthesis of mean differences between men and women on diverse psychological constructs supports this proposition (Hyde, 2005, 2014; Zell et al., 2015). We expect this to hold true across gender identity and sexual orientation without strong evidence to the contrary.

The second hypothesis is that differences might be more appropriately attributed to the social-structural systems rather than inherent, biological truths. In other words, differences often may not represent uniqueness to one group based on group affiliation but instead indicators of social-structural or systemic differences, like specific stresses that are experienced by members of a marginalized group, or having close, social communities in which different norms are used as reference points. It is not necessary that belonging to a group inherently creates these average 
differences; rather, experiences that are shaped by actual or perceived group membership may play a role.

Evidence to support the influence of social-structural systems on observed group differences comes from cross-cultural differences, cohort differences, and intervention research (Junkins et al., 2021; Lippa, 2009; Schmitt et al., 2017; Zell et al., 2015). Cross-cultural evidence shows that the gender ideologies of a nation relate strongly to mean differences in a nation between men and women (Schmitt et al., 2017). Cohort differences would be expected to be similar and persistent across generations across all time if the differences are inherent to group membership. However, this is not the case for gender differences related to gender normative behavior (Hsu et al., 2021), but it was the case when all differences were taken together and compared as average magnitude over the last several decades (Zell et al., 2015). Intervention research has successfully changed mean differences, at least in the short term, suggesting that differences are malleable and more strongly related to our experiences and socialization rather than inherent to group membership (Feng et al., 2007). Again, we expect this to remain true if we expand the gender similarities hypothesis to include gender identities beyond the binary and sexual orientation.

\section{Defining SGM Identities in Individual Differences Research}

One substantial challenge to comparing groups of people is the essentially limitless variety of identities that people may hold, either separately or simultaneously, including within the domains of gender and sexuality. Much of the psychological literature continues to collect and report participant gender or sexual identities as dichotomous between the most commonly, historical groups, such as male or female, gay or straight ([anonymized for review, 2022).

Beyond two-group comparisons, we can use more comprehensive categorical demographic 
questions with a self-identification option; this means for each demographic having more than two options listed (at least) and using text write-ins rather than an "other" option (Cameron \& Stinson, 2019). In addition to increasing agency and inclusion from the participant perspective, this type of grouping allows us to look at patterns across multiple, more specific groups.

The appeal of dichotomous approaches to group comparisons is often in the analytic simplicity. Informed by minority stress theory (Meyer, 2003), one option for a dichotomous approach to address broader categories explicitly splits people into SGM status and non-SGM status. SGM identities defined by National Institutes of Health include (but are not limited to) people who identify as other than heterosexual, have same-gender attraction, queer, Two-Spirit, transgender, nonbinary, intersex, and those who have nonbinary constructs of sexual orientation, gender, and/or sex (2021). The broad categorization of SGM has been used to frame the understanding of topics such as resilience (Meyer, 2007), psychotherapy (Budge et al., 2017), eating disorders (Nagata et al., 2020), and response to the COVID-19 pandemic (RodriguezSeijas et al., 2020). While stating SGM as a population of interest is parsimonious for researchers, such broad categorization may limit the potential for interpretation of variation within heterogeneous groups, and nuanced differences between identities cannot be uncovered at this level. It remains an open question whether participants are willing to classify their complex identities within such broad categories as SGM.

Dimensional approaches to measurement are often appealing for methodological and psychometric reasons, but standardized approaches to gender and sexuality have been elusive. Dimensional measures provide a way to look at differences in ways that categories cannot fully capture (Lippa, 2020), and represent differences in degree rather than differences in kind (Meehl, 1992). Therefore, the third way we propose to evaluate group differences between SGM 
identities is by examining the extent to which a dimensional sexuality measure recapitulates observed categorical differences in personality. There is support for this type of approach (Morales, 2020; Reis \& Carothers, 2014). Reis and Carothers (2014) examined established sex differences to determine if they reflect the existence of discrete groups or a continuum by running taxometric analyses on many datasets comprising many people and psychological indicators, finding that the data unambiguously in the majority of indicators resembled a dimension over taxonic models. In another study, Morales (2020) used a continuous measure of masculinity (agency) to estimate how much variation in group differences in HEXACO personality was related to masculinity. Of course, a requirement of taking a dimensional approach requires the availability of a continuous measure of the construct of interest. The measurement of sexuality, sexual orientation, and gender-related constructs is challenging with little consensus on ideal measurement (e.g., Ridner et al., 2007).

The most commonly used continuous measure of sexual orientation is the Kinsey scale (Kinsey et al., 2003), where participants rate themselves from zero (exclusive heterosexuality) to six (exclusive homosexuality). There is a lot of criticism around this approach (e.g., Hale et al., 2019; Zietsch \& Sidari, 2020) and ambiguity around the center point as being indicative of attraction to multiple genders, as in pansexual or bisexual identities, or none, as in asexual (Galupo et al., 2014; Walton et al., 2016). This additional dimension of sexuality not captured by the Kinsey scale is called fluidity, or the extent to which a person is not exclusively attracted to one gender. Thematic analyses of existing measures support the need for fluidity to be assessed and for the measures to be gender-inclusive (Galupo et al., 2014). The Epstein Sexual Orientation Inventory (ESOI) assesses both overall sexuality—referring more closely to sexual orientation — and sexual fluidity — meaning the range of sexual attraction to different genders 
(Epstein et al., 2012; Epstein \& Robertson, 2014). Figure 1 shows the average scores of different sexual orientation groups on these two dimensions. Taken together, we can estimate group differences by comparing two groups (SGM versus not), multiple categories of gender and sexual orientation, and a dimensional approach to estimate how much of the group differences are explained by a continuous measure related to the grouping.

Figure 1. Sexual Orientation Inventory plotted for five sexual orientations at their average level of sexuality (x-axis) and sexual fluidity (y-axis) in our data. The size of the circles are scaled by the sample size. There is a large overlap of the bisexual and pansexual circles as their averages were quite similar.

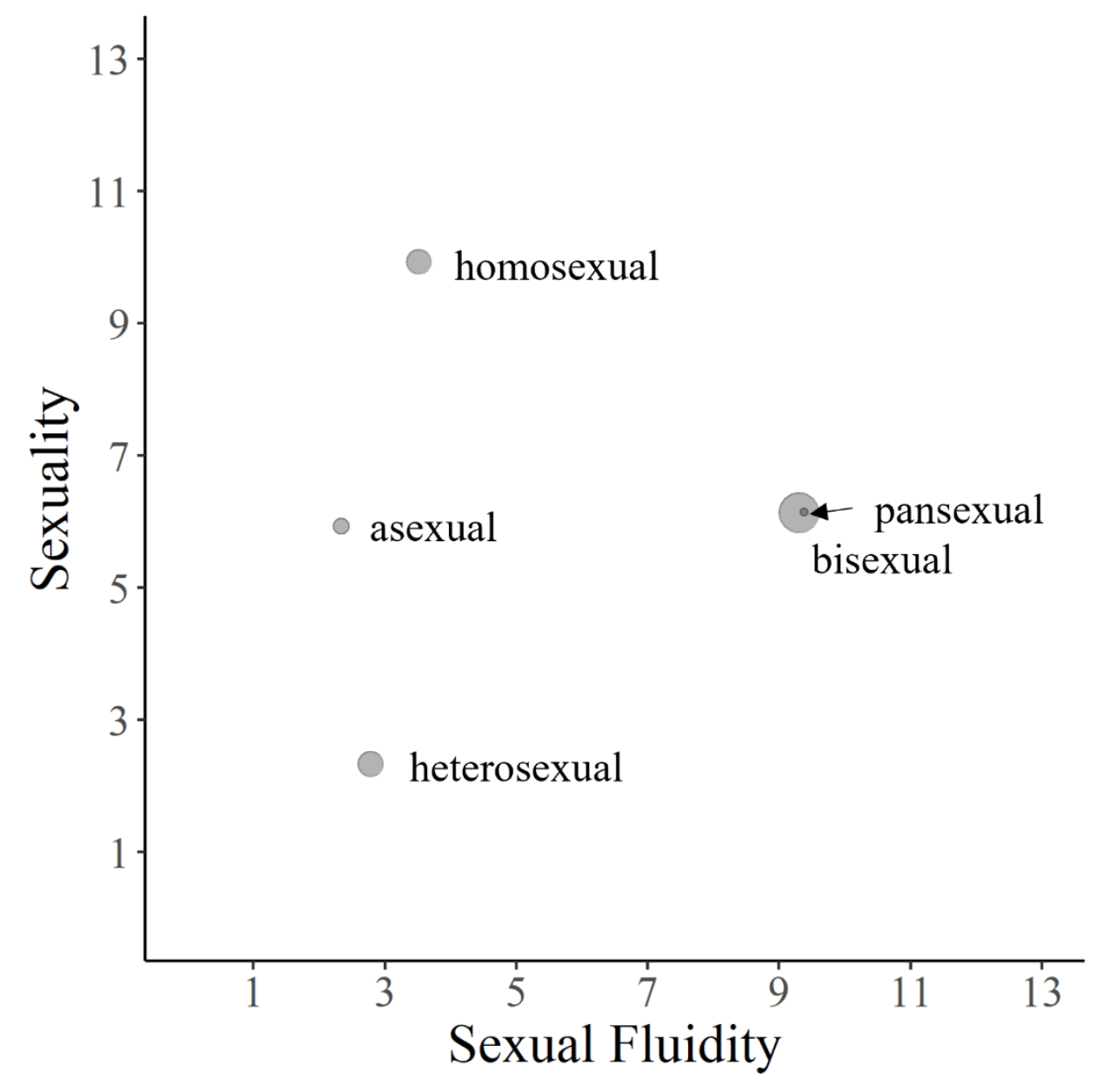




\section{Recruitment of SGM Participants via Social Media}

Research that is not geared to SGM populations at the outset tends to have demographic items that are not inclusive in psychology (Magliozzi et al., 2016), medical health literature (Cahill \& Makadon, 2014; Quinn et al., 2021), and national probability surveys (Federal Interagency Working Group, 2016a, 2016b). This lack of complete and transparent demographics yields a potentially severely biased view of the literature and may make federal agencies less effective at generating actions that address environmental justice in these underserved populations. The justification that it is difficult to recruit SGM groups is often asserted but rarely formally supported. In relationships research, this is commonly found to be a reason for only including heterosexual relationships without justifying the necessity of exclusion for the research question ([anonymized for review], 2022).

Online convenience methods have been shown to successfully recruit hard-to-reach populations. For example, one group used the Internet to reach more than 500,00 participants including those from low- and middle-income economies, non-Western societies, non-U.S., and non-college-aged respondents (Gosling et al., 2010). Social media platforms, like Reddit, present an opportunity to access established, relatively widespread, diverse participants' attention. Although these platforms, like college samples and survey sites, also are not representative of the general population, they afford greater public access than other recruitment methods. Reddit allows fast and free recruitment of targeted populations (Shatz, 2017), even compared to MTurk (Luong \& Lomanowska, 2021).

Direct comparisons between Reddit and undergraduate samples for replication of effects showed Reddit is distinct yet offers inexpensive, high-quality data (Jamnik \& Lane, 2017). Education, U.S. citizenship status, and college major were more diverse in the Reddit sample 
than in undergraduate samples (Jamnik \& Lane, 2017). Reddit sees approximately 52 million daily users (Dean, 2021). There is evidence that online research is not especially problematic compared to undergraduate research, MTurk, or Prolific (T. Buchanan \& Smith, 1999; Gosling et al., 2004). While social media platform recruitment is subject to the same limitations as all nonprobability sampling strategies, there are specific disciplines that especially benefit from online convenience methods, including work with marginalized communities (Catania et al., 2015).

\section{Current Study}

We expand on research investigating differences in Big Five factors among people grouped by sexual orientation and by gender using three approaches discussed earlier (dichotomous SGM status, diverse categorical, and dimensional) to test an expansion to the gender similarities hypothesis which we will refer to as the similarities hypothesis. To do so we collected a more diverse sample than prior personality research has reported by using online convenience recruitment methods to diversify the gender identities and sexual orientations represented. Self-identification allows us to describe whether there were other frequent patterns of gender or sexual orientation outside those we listed. In this way, we can look at group differences in personality traits and discuss trends among a greater diversity of sexual and gender identities.

The current study is largely descriptive and exploratory and, thus, we report effect sizes for mean differences and correlations among a diverse sample on the Big Five personality factors and facets and continuous sexuality measures. We do not expect large group differences $(d>.44$ based on the prior meta-analysis Allen \& Robson, 2020) to emerge across SGM vs non-minority, comparisons between sexual orientations collapsed across gender, or comparisons between 
gender identities collapsed across sexual orientation, with neuroticism and open-mindedness being the anticipated exceptions (Costa et al., 2001; Feingold, 1994; Zoeterman \& Wright, 2014). We have prioritized the following pre-registered questions:

How do personality factor and facet means differ across categorical indicators of gender and sexual orientation; and

Can a continuous measure of sexuality partially explain categorical sexual orientation group differences in personality?

We also pre-registered three additional analyses to evaluate data quality: evaluation of participant identification on a dichotomous question about SGM identity; measurement invariance of the personality measure across identity groups; and comparison of observed group differences to a previous, limited meta-analysis of community and clinical samples. Details for the data quality questions are presented in full in the online Supplemental materials. Preregistration, de-identified quantitative data, and analysis scripts are available at https://osf.io/kcqv8/?view_only=55bfc45eeeb144e696501c07c789dd38.

\section{Methods}

\section{Procedure}

The Institutional Review Board at University XXX approved this research under expedited review for no more than minimal risks to participants (IRB\#21508). Participants were recruited to an online Qualtrics survey via recruitment messages posted on Reddit, in the SampleSize subreddit (https://www.reddit.com/r/SampleSize/). Four posts were made on the subreddit from January to March 2021 (January 14, January 29, February 10, and March 4). Briefly, the recruitment messages read as "personality differences \& sexual orientation" and a 
one-sentence summary disclosing the purpose of the study read "the goal is not to make negative comparisons between groups but instead to update outdated research". Recruitment oversampled non-heterosexual and gender non-conforming participants through the recruitment message and framing of the research idea in the survey advertisement. There was no financial compensation for participation. Recruitment advertisement images are available in Supplemental Figures S2-3. A debrief was added at the end of the survey on January 15, 2021. By nature of how posts are displayed on Reddit, most participants completed the survey within the first 24 hours of an advertisement being posted (e.g., by January 15, 530 people had opened the link to the survey; see Supplemental Figure S2). The data were downloaded on April 5, 2021, after the preregistration of the analytic plan. In a separate form presented after the survey, 252 people provided an email address to receive a copy of the report when available, and the link to the preprint was sent to these participants on March 7, 2022. The mean time to complete the survey was 18.6 minutes $(S D=113.3$ minutes; 5 to $95 \%$ range $=4.7-26.3$ minutes $)$.

\section{Participants}

A total of 1,787 people followed the posted link to begin the survey. Following the plan for data preparation, participants who were younger than 18 were deleted $(N=17)$ and one inappropriate responder was deleted ${ }^{1}$. Participants with less than $75 \%$ of the total survey completed or $80 \%$ (or 12 items) of the personality inventory were excluded; the same 510 participants met both thresholds and thus were excluded ${ }^{2}$. The final sample was 1,259 people.

The mean age of participants was $M=24.52(S D=6.14$; range 18-61). Table 1 provides the participants' gender and sexual orientation. Participants were asked whether they self-

\footnotetext{
${ }^{1}$ The respondent put racial slurs in every open textbox.

${ }^{2}$ The average missingness for excluded participants was $88 \%$ of the full survey and $99.7 \%$ of personality items.
} 
identified as a sexual and/or gender minority and 72.6\% responded yes (see Table S0). 52.8\% of participants reported their country of residence to be the United States, with 55 countries of residence reported across all participants. The self-description of race and ethnicity from one textbox was coded in two ways (see Table S3 and Figure S1). Using the classifications from the United States Census Bureau, $80 \%$ of participants identified as White, $6 \%$ as Asian, $2 \%$ as Black, $0.8 \%$ Native or Indigenous persons or Pacific Islander, and 2\% as multiracial. Six percent indicated Hispanic or Latinx ethnicity. $63.7 \%$ of participants reported some post-secondary education. Forty-four percent of the participants were currently employed and $48 \%$ reported being students; $50.6 \%$ reported their relationship status as single. See Supplement for more demographic details as well as coding decisions for gender identity and sexual orientation text responses.

Table 1. Count of participants by gender and sexual orientation.

\begin{tabular}{|c|c|c|c|c|c|c|c|c|c|c|}
\hline Gender & & & 2 & Sexual orien & ation & & & & & \\
\hline & Asexual & Bisexual & Pansexual & Homosexual & Heterosexual & Queer & Unsure & $\begin{array}{l}\text { All } \\
\text { other }\end{array}$ & NA & Total \\
\hline $\begin{array}{l}\text { Cisgender } \\
\text { man }\end{array}$ & 34 & 80 & 16 & 70 & 120 & 0 & 18 & 4 & 2 & 354 \\
\hline $\begin{array}{c}\text { Transgender } \\
\text { man }\end{array}$ & 8 & 36 & 6 & 16 & 4 & 3 & 2 & 2 & 0 & 77 \\
\hline $\begin{array}{c}\text { Cisgender } \\
\text { woman }\end{array}$ & 76 & 238 & 48 & 91 & 88 & 10 & 4 & 11 & 1 & 567 \\
\hline $\begin{array}{c}\text { Transgender } \\
\text { woman }\end{array}$ & 2 & 12 & 7 & 14 & 0 & 0 & 0 & 1 & 0 & 36 \\
\hline Nonbinary $^{3}$ & 25 & 55 & 39 & 16 & 2 & 11 & 4 & 16 & 0 & 168 \\
\hline All other & 9 & 12 & 12 & 7 & 3 & 2 & 3 & 7 & 0 & 55 \\
\hline NA & 0 & 0 & 0 & 0 & 1 & 0 & 1 & 0 & & 2 \\
\hline Total & 154 & 433 & 128 & 214 & 218 & 26 & 32 & 51 & 3 & \\
\hline
\end{tabular}

\section{Measures}

\footnotetext{
${ }^{3}$ One person identified as "transgender nonbinary" and is included with all other nonbinary participants.
} 
Demographics included age, gender, race (text entry), country, employment, relationship status, relationship type, and sexual orientation. Man, woman, and nonbinary were collapsed across cisgender and transgender (e.g., cisgender women and transgender women are women), as previous research demonstrates that individual patterns of response among transgender participants mirror patterns observed in same-gender cisgender participants (Olson et al., 2015) and because of the low number of responses. Race, country, employment, relationship status, and relationship type were collected to describe our sample but were not included in analyses.

Text fields. For gender identity and sexual orientation, there was a write-in response option, and we coded these responses keeping closely to the participant's words. For this reason, we did not code self-identifications into any existing categorical choices unless an adjective or synonym was given $\left(n=12^{4}\right.$; e.g., "male" instead of cisgender man, "MTF" instead of transgender woman). No gender self-identifications reached a sample size greater than 20, therefore coded as "all other". For sexual orientation, we coded text responses into existing categories if participants specified their romantic orientation (e.g., asexual heteroromantic would be coded as asexual; $n=8 ; 1$ participant was added to "unsure"). Only the self-identification of "queer" reached the threshold for defining an additional category $(n=26)$.

Big Five Inventory-2. The BFI-2 is a 60-item inventory assessing five factors: extraversion (facets: sociability, assertiveness, and energy level), agreeableness (compassion, respectfulness, and trust), open-mindedness (intellectual curiosity, aesthetic sensitivity, and creative imagination), conscientiousness (organization, productiveness, and responsibility), and negative emotionality (anxiety, depression, and emotional volatility). The items are brief

\footnotetext{
4 One participant identified as "transgender nonbinary", so we added to nonbinary category so as not to reveal too much in crosstabulations about one individual.
} 
definitions, adjectives, or synonyms of traits (i.e., "Is complex, a deep thinker.") that participants will respond 1 (disagree strongly) to 5 (agree strongly) that they are "someone who...". The scale also allows for the scoring of 15 narrower facets within the Big Five factors with four items apiece (Soto \& John, 2017). Factor-level and facet-level averages were obtained from the corresponding items.

Sexual Orientation Inventory. The ESOI is an 18-item measure assessing sexual orientation on two continuous dimensions. Half of the items measure same-sex fantasies and behaviors and the other half, opposite-sex fantasies and behaviors. For same-sex (SS) and opposite-sex (OS) interactions, items are assessed 0 (no) and 1 (yes), or a 0 (not at all, never have them), 1 (moderately, rare or occasional), and 2 (very strongly, frequent) to obtain the raw SS score and raw OS score. The raw OS score is then reverse-scored and averaged with the raw SS score to calculate the mean sexuality. The result is higher scores relate to homosexuality on the continuum and lower scores relate to heterosexuality. The range of sexuality, measuring the fluidity of sexual fantasies and behaviors, is based on the lower raw score (Epstein et al., 2012; Epstein \& Robertson, 2014). For example, someone with a raw OS (heterosexuality) score of 6 and a raw SS (homosexuality) score of 8 , has a lower OS score; their fluidity is a 6. Average sexuality and sexual fluidity, calculated as described, were used in analyses.

Sexual and/or Gender Minority Label. Participants responded "yes" or "no" to whether they "consider [themselves] a member of a sexual and/or gender minority?". We randomly presented the question alongside the NIH definition of a SGM (National Institutes of Health, 2021) or simply the question itself with no definition. The NIH definition for someone who is a sexual and/or gender minority: 
“includes, but is not limited to, individuals who identify as lesbian, gay, bisexual, asexual, transgender, Two-Spirit, queer, and/or intersex. Individuals with same-sex or gender attractions or behaviors and those with a difference in sex development are also included. These populations also encompass those who do not self-identify with one of these terms but whose sexual orientation, gender identity or expression, or reproductive development is characterized by non-binary constructs of sexual orientation, gender, and/or sex." (National Institutes of Health, 2021)

\section{Statistical Analyses}

All analyses were performed in R (Version 3.6.1, R Core Team, 2019). Scripts and materials are available here to reproduce all reported results:

https://osf.io/kcqv8/?view_only=55bfc45eeeb144e696501c07c789dd38. Full data is not openly accessible, because of the sensitive text responses and concerns for privacy and confidentiality. The personality and sexuality scale items are available with no demographic data.

Missingness. Participants who completed less than $75 \%$ of the total survey questionnaire, and $80 \%$ of the 60 personality items, were excluded. According to Little's MCAR test, which states under the null hypothesis that there are no differences between the grand means of different missing-value patterns, from the naniar R package (Tierney et al., 2021), we concluded the data was missing completely at random $\left(\chi^{2}(4780)=3591.63, p=1\right)$.

Statistical power analyses. A priori power analyses were undertaken to estimate sample sizes needed for each research question below; details are provided in the Supplemental Materials. The $\mathrm{R}$ packages proportion, $p w r$, and paramtest were used to calculate a priori statistical power (Champely, 2020; Hughes, 2017; Subbiah \& Rajeswaran, 2017). Small, 
medium, and large effect sizes were defined based on the distribution of effect sizes observed in Allen and Robson's (2020) meta-analysis. A small effect was thus defined as Cohen's $d$ of 0.22 , medium $d=.33$, and large $d=.44$. On average, we require $N>200$ for $90 \%$ power to detect medium Cohen's $d$ and $N>250$ for a correlation coefficient $(r)$ of 0.20 . This is similar to the threshold of $N>250$ suggested by Schönbrodt \& Perugini (2013) as a reasonable sample size at which correlations are expected to stabilize. Confidence intervals $(95 \%)$ are reported alongside all effect size estimates. For those groups that fall below our minimum thresholds $(\sim 200$ participants), we do not expect to achieve reliable estimates that bear strong interpretation. We planned to report all the exploratory results; however, the power analyses determined our standards for interpreting patterns of differences.

Descriptive Statistics. We reported basic descriptive statistics, effect sizes, exact pvalues, and 95\% confidence (credible) intervals. Distributions of the sexuality measures were displayed, including group differences, in the online OSF materials. The correlations among study variables were computed using Pearson's correlation coefficient.

Data Quality Analyses. We first sought to evaluate the quality of the data obtained from our survey process in three ways, described in full in the Supplemental Materials. We first characterized the extent to which participants were willing to self-identify with the 'SGM' label by randomly assigning participants to either view the NIH definition, or not, and evaluating whether the viewing of the definition changed the proportion of participants identifying as SGM. We next evaluated measurement equivalence of the Big Five personality traits across the categories defined below, fitting multi-group confirmatory factory analysis models to determine 
multiple-group invariance with categorical outcomes ${ }^{5}$ following theory-driven personality models, with no data-driven improvements (Svetina et al., 2020; Wu \& Estabrook, 2016; see details in Supplemental Methods). To run a multi-group confirmatory factor analysis, we used Rpackages lavaan (Rosseel, 2012) and semTools (Jorgensen et al., 2021). To have maximum comparability to the other samples, we used average scores to estimate personality differences in all other analyses. Finally, we compared our sample means, variances, and mean differences between limited groups (those represented in the previous literature) with existing estimates, to evaluate whether our more diverse, convenience sample was consistent with previously described samples.

Mean Differences Between Groups. Mean differences between groups were calculated using Cohen's $d$ for personality traits and facets using the R-package rstatix (Kassambara, 2021). Based on our power analyses described above, we had $90 \%$ power to detect large effect sizes given groups $>100$, medium effect sizes given groups $>200$, and small effect sizes $(d=0.22)$ given groups $\geq 450$. At the minimum sample size threshold $(N>100)$, we interpreted large effect sizes and reported patterns among small to medium effect sizes. Results along with confidence intervals are reported in the Supplemental Tables S10-13.

Saturation Analysis with a Continuous Measure. To evaluate whether the pattern of differences across groups could be accounted for by a dimensional measure of sexuality, we conducted a saturation analysis. As described by Morales (2020), the mean differences (Cohen's d) between sexual orientation categories and personality facets were saved. Then, correlations between the continuous sexuality measure and personality facets were saved. The specific groups

\footnotetext{
${ }^{5}$ We preregistered that we would use steps laid out by Meredith (1993), but the data are more appropriately treated as categorical, not continuous.
} 
for comparison were limited to those that met the minimum sample threshold of at least 250 participants, so heterosexual, bisexual, and homosexual group comparisons were included. The correlation of these two columns of $15 \times 3$ (facets $\mathrm{x}$ sexual orientations compared) rows was calculated. We estimated this procedure twice, once for group differences explained by overall sexuality measure and once for differences explained by sexual fluidity. This correlation, or saturation, represents the ability of the sexuality measure to explain the differences in personality observed between sexual orientation groups.

\section{Results}

The correlations among study variables and descriptive statistics are reported in Tables S4-5. As presented in the Supplemental Materials, evaluation of data quality demonstrated that although participants were more likely to overall self-identify as SGM when presented with the $\mathrm{NIH}$ definition this difference does not appear to be due to any limited number of identity groups consistently self-excluding. Measurement invariance of the personality measure held broadly across groups. Mean differences between our current sample and the derivation sample for the personality measure (Soto, 2019) were consistently present. Standard deviations on personality factors and facets were also larger in our sample compared to Soto (2019), consistent with an interpretation of a more diverse sample. However, our within-sample estimates of group differences were highly consistent with the limited group differences that have previously been reported ( $r=0.741$, Allen \& Robson, 2020). This suggests that our data and results are consistent with, but more diverse than, previously investigated samples.

\section{Mean Differences Between Groups}


We computed standardized mean differences between SGM and non-SGM participants, gender categories (nonbinary—men—women), and sexual orientation categories (asexual— bisexual—heterosexual—homosexual—pansexual)(see Figure 2; Table S10-13).

Figure 2. Personality trait means plotted by identity (sexual and gender) with standard deviations shown as shaded regions to show the variability in the current sample.
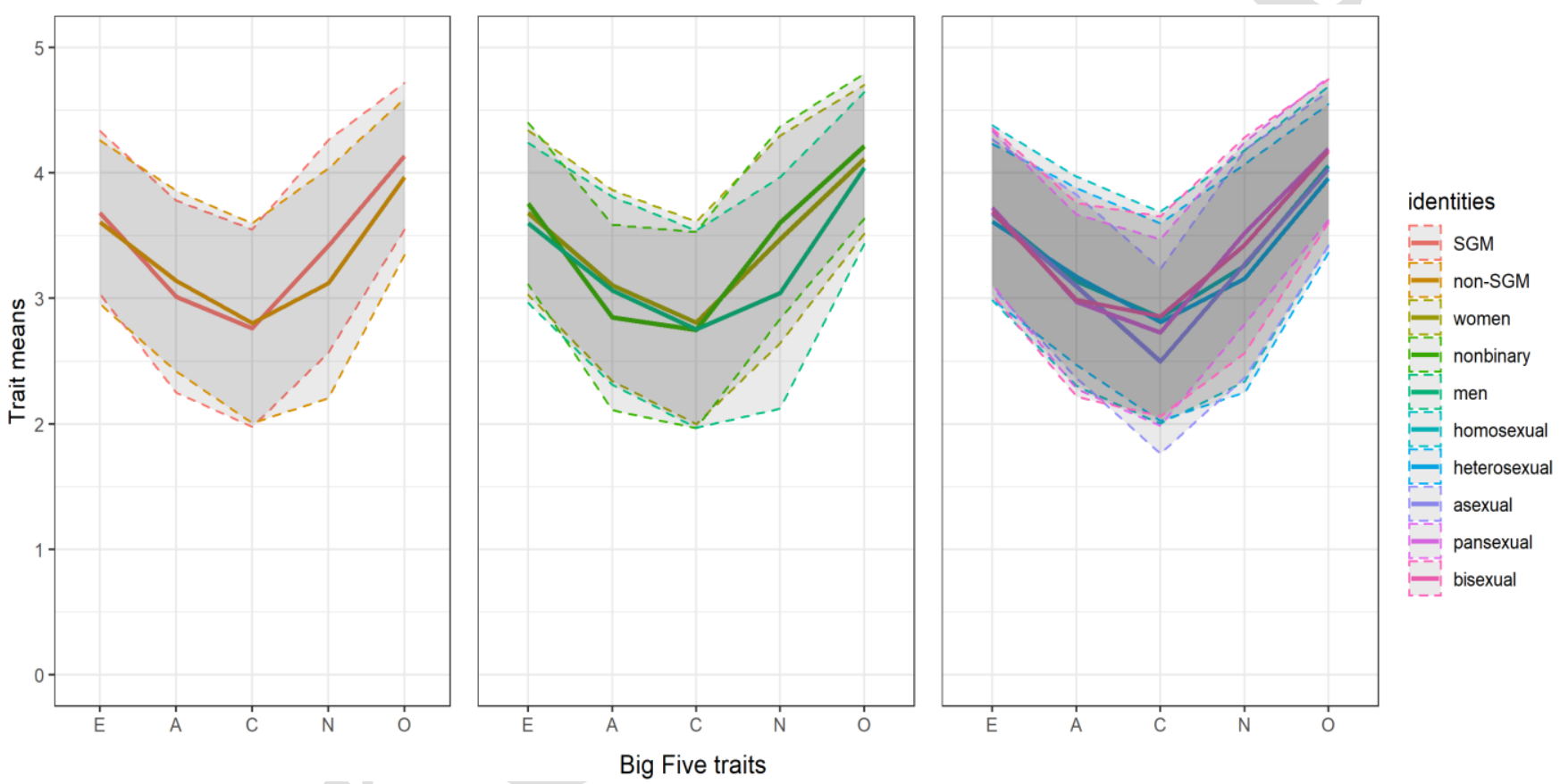

For SGM versus non-minority participants, there were no large differences as we hypothesized. Non-SGM participants reported higher conscientiousness $(d=0.17,95 \% \mathrm{CI}[.05$, .30]) than SGM participants, and SGM participants reported higher levels of neuroticism (-0.34 $[-.46,-.20])$ and open-mindedness $(-0.28[-.40,-.16])$. There were no differences in extraversion or agreeableness (all $|d|<0.12)$.

For gender identities, mean differences at the personality factor level manifested for all but extraversion among any two groups. There were large observed differences in neuroticism between nonbinary people and men $(d=0.66,95 \% \mathrm{CI}[.49, .85])$ and men and women $(-0.49$ [- 
$.62,-.37])$. While there were small to medium differences in open-mindedness between nonbinary people and men $(0.30[.10, .48])$ and women $(0.18[.02, .36])$, aesthetic sensitivity is what differed the most between nonbinary participants and men $(0.60[.43,79])$ and less so with women $(0.28[.12, .45])$. Nonbinary participants and women were similar in neuroticism. Broadly, nonbinary participants reported lower conscientiousness, higher neuroticism, and higher open-mindedness than men; women reported higher neuroticism and slightly higher agreeableness than men.

For sexual orientation, mean personality factor differences existed for all but agreeableness among any two groups. Open-mindedness and neuroticism differed the most among groups as expected. Asexual participants reported lower extraversion compared to any other group $(|d|=0.31-0.47)$. Generally, bisexual and pansexual participants did not greatly differ from one another and neither did heterosexual and homosexual participants. The pattern of effects between bisexual and pansexual as well as heterosexual and homosexual participants differed in magnitude. Heterosexual participants were more different from bisexual or pansexual participants than homosexual participants. The pattern observed for asexual identities was that they were more similar to heterosexual and homosexual than bisexual or pansexual participants.

Across all levels of defining categorical groups, the average effect size was 0.16 , which is small; no observed difference was greater than 0.66 standard deviation units between any two groups. Most effect sizes $(73 \%)$ fell in the range of $0<|d| \leq 0.22$ referring to nonexistent to small effects. The observed patterns were in line with expectations: first, that neuroticism and open-mindedness would differ the most and, second, that groups were more similar than different generally. Taken together, the evidence of the size of our mean differences supports the 
first proposition of the similarities hypothesis, referring to most differences being small, few large differences, and these few large differences not existing systematically.

\section{Saturation Analysis with a Continuous Measure}

Since we calculated two variables from the sexual orientation inventory items (sexuality and fluidity), we estimated a saturation analysis separately for each variable. First, we estimated how much the sexuality scale can explain personality differences between sexual orientation groups that met the specified sample thresholds. The sexuality saturation was $r=0.301$, $95 \% \mathrm{CI}[0.008,0.546]$ with sexual orientation group differences in personality facets, which suggests that a continuous measure of sexuality does moderately explain the differences between groups observed. Second, we ran the same procedure for sexual fluidity. The sexual fluidity saturation was $r=0.452,95 \% \mathrm{CI}[0.182,0.658]$ with sexual orientation group differences in personality facets, which suggests again we are explaining more of the differences. However, these saturations mean that only $9-20 \%$ of the variation in personality differences is explained by these dimensions of sexuality. If we sought to attribute these group differences to their sexuality, then this might suggest that this would be inappropriate. Instead, this supports the similarities hypothesis that attributions should be made to contextual factors or other third variables.

\section{Discussion}

The results from our research questions support an expanded similarities hypothesis as applied to personality factors and facets. The hypothesis states that people, when categorized by their gender identity or sexual orientation, are more alike than different on most psychological variables. It further suggests that causes or explanations for differences when they do emerge 
might be placed on social-structural systems or larger cultural forces, rather than assuming inherent group membership causes these differences. Using the current sample of participants, of whom $72.6 \%$ self-identified as members of a sexual and/or gender minority, we sought to establish the utility of online convenience methods to enhance the diversity of psychological science, and improve the representation of understudied identities (e.g., nonbinary, asexual, pansexual participants) in personality psychology, specifically. Although measurement invariance of the personality measure held broadly across groups, mean differences between our current sample and the derivation sample for the personality measure (Soto, 2019) were consistently present. Standard deviations on personality factors and facets were also larger in our sample compared to Soto (2019), consistent with an interpretation of a more diverse sample. However, our within-sample estimates of group differences were highly consistent with the limited group differences that have previously been reported ( $r=0.741$, Allen \& Robson, 2020). Overall, in support of an expansion of the similarities hypothesis, group differences in personality between gender identities and sexual orientations are uncommon and unsubstantial, regardless of how groups are defined.

\section{Expanding the Similarities Hypothesis}

We found there were small average differences $(d=0.16)$ in personality factors and facets between any two groups. This result was in line with previous research on personality between men and women (Hyde, 2005), multiple psychological domains between men and women (Zell et al., 2015), and personality between sexual orientation groups (Allen \& Robson, 2020). However, we expanded the results to include namely nonbinary, pansexual, and asexual people, seeking to expand the similarities hypothesis to include more diverse gender identities and sexual orientations. 
Saturation analyses suggested that a continuous measure of sexuality captures $r^{2}=9 \%$ of the personality differences among categorical sexual orientation identities, while sexual fluidity can explain $r^{2}=20 \%$ of the personality differences among categorical sexual orientation identities. These results are purely descriptive and may be limited by the items used to measure sexuality via thoughts, feelings, and behavior toward members of the 'same' versus 'opposite' sex, which implies a binary of both sex and gender. Whereas sexual fluidity was more strongly correlated with the differences than sexuality, this may be due to the inclusion of only monosexual (i.e., heterosexual and homosexual) versus bisexual participants. As we saw, monosexual participants, or those mainly attracted to one sex, were more similar. Yet, the current continuous measure of sexuality alone cannot explain why members of different groups show average differences in personality supporting the argument that other contextual factors are relevant to attributions for differences.

\section{Data Quality and Limitations}

Specific strengths of this research were the diverse, largely SGM-identified sample, the availability of a personality inventory that allowed evaluation of both broad factors as well as narrow facets, and measurement equivalence testing. A finer-grained comparison of personality identified some cases where a single facet appeared responsible for an average factor difference. Full reporting of the exploratory analyses conducted here will allow future research to develop narrower hypotheses and rule out the plausibility of certain hypotheses, including the idea that group differences may be large and pervasive. The Supplement provides a discussion of the write-in responses provided by participants and suggestions for inclusive recruitment and tailoring the demographic questions to fit study purposes. In short, two effective strategies were 1) being clear, upfront, and engaged with respondents about the research purpose, and 2) 
including more appropriate categories of gender and sexual orientation plus a write-in option that requires cleaning. Diversifying the samples included in personality psychology is in and of itself a strength because it produces more broadly applicable knowledge (Cornesse et al., 2020; Mook, 1983).

The recruitment strategy on Reddit (r/SampleSize) relied on people's interests in surveys, in personality and sexuality, and inclusive science. We were responsive and transparent with respondents. In the end, 252 people out of the 1,259 participants provided an email to receive a copy of the written report. The sample demographics, the high responses in short periods of time, and the level of engagement all suggest our strategy was effective at getting more diverse respondents along targeted axes of diversity. When we compared some of our mean differences to a recent meta-analysis of personality differences, we found there was a strong positive correlation $(r=0.74)$ and we passed Q-tests for homogeneity the majority of the time, suggesting our effects were mostly similar to the meta-analyzed pooled mean differences. These results, combined with the larger differences between our sample and a U.S. nationally representative adult sample, suggest that our Reddit sample was different from the national U.S. population but less different from samples sampled to have more diversity of sexual orientations. These comparisons helped to establish who our sample was and the validity of the sampling strategy.

Personality facets demonstrated measurement variance across varying categorical groupings of sexual orientation and gender. This result suggests that people responded to items equivalently allowing for metrics to be directly compared. Measurement invariance along these dimensions of diversity bolsters the psychometric properties and validity of the BFI-2. We were limited in our ability to estimate measurement invariance and means (and differences) for several specific SGM identities by small group sample sizes. For example, measurement invariance was 
not tested for transgender people, because of low sample size. Instead, they were collapsed with men, women, or nonbinary as appropriate. Further, measurement invariance was tested using two non-ideal approaches because a small number of items did not have endorsement of all responses within each group, which could be seen either as a concern for the measurement tools or a reason for using multiple sampling strategies. Bogaert et al. (2018), also, found measurement invariance of HEXACO personality within-gender between-orientations. Although we achieved sample sizes for specific SGM identities that are far greater than is typically available (or reported) in psychological research, our sample did not allow for comparisons among many of the less frequently reported categorical groups (e.g., queer sexual orientation), or between categorical groups defined by both gender and sexual orientation (e.g., asexual women).

One limitation of the current study is that it sampled narrowly from one forum within one social media site. Approximately $18 \%$ of U.S. adults say they use Reddit, compared to Twitter at 23\% (Auxier \& Anderson, 2021). Further breaking down U.S. estimates, 23\% of adult men, 12\% adult women, $17 \%$ White, $17 \%$ Black, 14\% Hispanic, 48\% 18-29-year-olds, $29 \%$ income of \$30k-50k, 24\% some college, and 24\% urban adults use Reddit. According to Pew Research Center, Reddit is one of the few growing social media platforms (Auxier \& Anderson, 2021). We are not the first to name Reddit as a source for convenient, high-quality data (e.g., Jamnik \& Lane, 2017); however, we focus here on the utility of sites like Reddit to specifically attain better diversity for hard-to-reach populations in our samples. There are concerns relevant to online research in general and to using one singular social platform; however, research suggests there are not generally large differences between participants filling a survey online versus offline ( $\mathrm{T}$. Buchanan \& Smith, 1999; Riva et al., 2003). The sampling approach also differs from the prior studies, such as the meta-analysis and the representative U.S. sample used as points of 
comparison (Allen \& Robson, 2020; Soto, 2019), as we used no country restrictions, age restrictions, college versus community restrictions, or the like. One solution to evaluate the generalizability of convenience samples is to replicate the research with multiple samples (e.g., McEwan, 2020; Peterson \& Merunka, 2014). We sought to provide a detailed demographic profile of the participants included in this study to improve the ability to compare characteristics with other samples in the future.

A second potential limitation was the use of inclusive language as part of the recruitment strategy which could have invited selection bias for either very excited people or skeptical people. While the feedback from participants was overwhelmingly positive, we cannot rule out selection bias based on a like or dislike of an emphasis on inclusive science. The timing of the recruitment, also, may influence observed effects that could differ across time and place; increased recognition of unfair practices and unequal treatment directed at BIPOC and LGBTQIA+ people represents a shifting social context that can affect both participant recruitment and perhaps even actual individual trait levels. Overall, these descriptive results demonstrate that the recruitment of SGM participants to psychological research is feasible.

\section{Conclusions and Future Directions}

In summary, we provided evidence to support and expand the similarities hypothesis for personality, at least, that people based on gender or sexual orientation are more alike than different. Importantly, measurement equivalence was supported; therefore, we show evidence that measures developed and normed on affluent Western undergraduate samples were capturing similar latent factors across diverse gender and sexuality identities (Lewis, 2021). Future research can track how mean differences change over time as culture changes and estimate how third variables representing sociocultural structures are related to mean differences. Additionally, 
other assumptions of "differences" can be viewed under a similarities hypothesis that contextualizes effect sizes measuring differences and values multiculturalism.

We, also, show that online convenience sampling is one way to increase sample diversity with regard to diverse sexualities and genders. Even though internet research is fallible (like all other commonly used recruitment strategies) in terms of other non-representative or validity concerns, a recent survey in the U.S. estimated that $12-14 \%$ of adults identify as LGBTQIA+ (Anderson et al., 2021). In comparison, the current sample was composed of $72.6 \%$ SGMidentifying people. Special considerations should be made before generalizing or assuming representativeness, but in our view, the benefits of greater opportunity for the inclusion of diverse participants in psychological research outweigh the costs. We recommend improving sampling demographics, using internet studies with large sample sizes, and duplicating in smaller community samples using targeted recruitment for applied research. Rather than penalizing internet research, we should develop better reporting standards for its use and encourage empirical evaluations of its validity (e.g., Shatz, 2017). By providing participants with the opportunity to describe themselves, psychological research can accurately describe and improve its inclusion of marginalized sexual orientations and gender identities.

\section{References}

Allen, M. S., \& Robson, D. A. (2020). Personality and Sexual Orientation: New Data and Metaanalysis. The Journal of Sex Research, 57(8), 953-965. https://doi.org/10.1080/00224499.2020.1768204

Anderson, L., File, T., Marshall, J., McElrath, K., \& Scherer, Z. (2021). New Household Pulse Survey Data Reveals Differences between LGBT and Non-LGBT Respondents During 
COVID-19 Pandemic (Household Pulse Survey, July - September 2021). U.S. Census Bureau.

Auxier, B., \& Anderson, M. (2021). Social Media Use in 2021. Pew Research Center. https://www.pewresearch.org/internet/2021/04/07/social-media-use-in-2021/

Batz-Barbarich, C., Tay, L., Kuykendall, L., \& Cheung, H. K. (2018). A Meta-Analysis of Gender Differences in Subjective Well-Being: Estimating Effect Sizes and Associations With Gender Inequality. Psychological Science, 29(9), 1491-1503. https://doi.org/10.1177/0956797618774796

Bogaert, A. F., Ashton, M. C., \& Lee, K. (2018). Personality and Sexual Orientation: Extension to Asexuality and the HEXACO Model. The Journal of Sex Research, 55(8), 951-961. https://doi.org/10.1080/00224499.2017.1287844

Buchanan, N. T., Perez, M., Prinstein, M. J., \& Thurston, I. B. (2021). Upending racism in psychological science: Strategies to change how science is conducted, reported, reviewed, and disseminated. American Psychologist, 76(7), 1097-1112. https://doi.org/10.1037/amp0000905

Buchanan, T., \& Smith, J. L. (1999). Using the Internet for psychological research: Personality testing on the World Wide Web. British Journal of Psychology, 90(1), 125-144. https://doi.org/10.1348/000712699161189

Budge, S. L., Israel, T., \& Merrill, C. R. S. (2017). Improving the lives of sexual and gender minorities: The promise of psychotherapy research. Journal of Counseling Psychology, 64(4), 376-384. https://doi.org/10.1037/cou0000215 
Cahill, S., \& Makadon, H. (2014). Sexual Orientation and Gender Identity Data Collection in Clinical Settings and in Electronic Health Records: A Key to Ending LGBT Health Disparities. LGBT Health, 1(1), 34-41. https://doi.org/10.1089/lgbt.2013.0001

Cameron, J. J., \& Stinson, D. A. (2019). Gender (mis)measurement: Guidelines for respecting gender diversity in psychological research. Social and Personality Psychology Compass, 13(11). https://doi.org/10.1111/spc3.12506

Catania, J. A., Dolcini, M. M., Orellana, R., \& Narayanan, V. (2015). Nonprobability and Probability-Based Sampling Strategies in Sexual Science. The Journal of Sex Research, 52(4), 396-411. https://doi.org/10.1080/00224499.2015.1016476

Champely, S. (2020). pwr: Basic Functions for Power Analysis. https://CRAN.Rproject.org/package $=$ pwr

Cochran, S. D., \& Mays, V. M. (2009). Burden of psychiatric morbidity among lesbian, gay, and bisexual individuals in the California Quality of Life Survey. Journal of Abnormal Psychology, 118(3), 647-658. https://doi.org/10.1037/a0016501

Committee on Understanding the Well-Being of Sexual and Gender Diverse Populations, Committee on Population, Division of Behavioral and Social Sciences and Education, \& National Academies of Sciences, Engineering, and Medicine. (2020). Understanding the Well-Being of LGBTQI+ Populations (C. J. Patterson, M.-J. Sepúlveda, \& J. White, Eds.; p. 25877). National Academies Press. https://doi.org/10.17226/25877

Cornesse, C., Blom, A. G., Dutwin, D., Krosnick, J. A., De Leeuw, E. D., Legleye, S., Pasek, J., Pennay, D., Phillips, B., Sakshaug, J. W., Struminskaya, B., \& Wenz, A. (2020). A Review of Conceptual Approaches and Empirical Evidence on Probability and 
Nonprobability Sample Survey Research. Journal of Survey Statistics and Methodology, 8(1), 4-36. https://doi.org/10.1093/jssam/smz041

Costa, P. T., Terracciano, A., \& McCrae, R. R. (2001). Gender differences in personality traits across cultures: Robust and surprising findings. Journal of Personality and Social Psychology, 81(2), 322-331. https://doi.org/10.1037/0022-3514.81.2.322

Dean, B. (2021). Reddit User and Growth Stats. BACKLINKO. https://backlinko.com/redditusers

Epstein, R., McKinney, P., Fox, S., \& Garcia, C. (2012). Support for a Fluid-Continuum Model of Sexual Orientation: A Large-Scale Internet Study. Journal of Homosexuality, 59(10), 1356-1381. https://doi.org/10.1080/00918369.2012.724634

Epstein, R., \& Robertson, R. E. (2014). How to Measure Sexual Orientation Range and Why It's Worth Measuring. Journal of Bisexuality, 14(3-4), 391-403. https://doi.org/10.1080/15299716.2014.933378

Federal Interagency Working Group. (2016a). Current Measures of Sexual Orientation and Gender Identity in Federal Surveys. (Improving Measurement of Sexual Orientation and Gender Identity in Federal Surveys.). https://nces.ed.gov/FCSM/pdf/buda5.pdf

Federal Interagency Working Group. (2016b). Toward a Research Agenda for Measuring Sexual Orientation and Gender Identity in Federal Surveys: Findings, Recommendations, and Next Steps. (Improving Measurement of Sexual Orientation and Gender Identity in Federal Surveys.). https://nces.ed.gov/FCSM/pdf/SOGI_Research_Agenda_Final_Report_20161020.pdf Feingold, A. (1994). Gender differences in personality: A meta-analysis. Psychological Bulletin, 116(3), 429-456. https://doi.org/10.1037/0033-2909.116.3.429 
Feinstein, B. A., \& Dyar, C. (2017). Bisexuality, Minority Stress, and Health. Current Sexual Health Reports, 9(1), 42-49. https://doi.org/10.1007/s11930-017-0096-3

Feng, J., Spence, I., \& Pratt, J. (2007). Playing an Action Video Game Reduces Gender Differences in Spatial Cognition. Psychological Science, 18(10), 850-855. https://doi.org/10.1111/j.1467-9280.2007.01990.x

Galupo, M. P., Mitchell, R. C., Grynkiewicz, A. L., \& Davis, K. S. (2014). Sexual Minority Reflections on the Kinsey Scale and the Klein Sexual Orientation Grid: Conceptualization and Measurement. Journal of Bisexuality, 14(3-4), 404-432. https://doi.org/10.1080/15299716.2014.929553

Gosling, S. D., Sandy, C. J., John, O. P., \& Potter, J. (2010). Wired but not WEIRD: The promise of the Internet in reaching more diverse samples. Behavioral and Brain Sciences, 33(2-3), 94-95. https://doi.org/10.1017/S0140525X10000300

Gosling, S. D., Vazire, S., Srivastava, S., \& John, O. P. (2004). Should We Trust Web-Based Studies? A Comparative Analysis of Six Preconceptions About Internet Questionnaires. American Psychologist, 59(2), 93-104. https://doi.org/10.1037/0003-066X.59.2.93

Haas, A. P., Eliason, M., Mays, V. M., Mathy, R. M., Cochran, S. D., D’Augelli, A. R., Silverman, M. M., Fisher, P. W., Hughes, T., Rosario, M., Russell, S. T., Malley, E., Reed, J., Litts, D. A., Haller, E., Sell, R. L., Remafedi, G., Bradford, J., Beautrais, A. L., ... Clayton, P. J. (2010). Suicide and Suicide Risk in Lesbian, Gay, Bisexual, and Transgender Populations: Review and Recommendations. Journal of Homosexuality, 58(1), 10-51. https://doi.org/10.1080/00918369.2011.534038 
Hale, A., Miller, L. B., Weaver, J., Husney, S. Q., \& Henares, R. (2019). The Dual Scales of Sexual Orientation. Journal of Bisexuality, 19(4), 483-514. https://doi.org/10.1080/15299716.2019.1650318

Harden, K. P., \& Koellinger, P. D. (2020). Using genetics for social science. Nature Human Behaviour, 4(6), 567-576. https://doi.org/10.1038/s41562-020-0862-5

Henrich, J., Heine, S. J., \& Norenzayan, A. (2010). Most people are not WEIRD. Nature, 466(7302), 29-29. https://doi.org/10.1038/466029a

Hsu, N., Badura, K. L., Newman, D. A., \& Speach, M. E. P. (2021). Gender, “masculinity,” and "femininity": A meta-analytic review of gender differences in agency and communion. Psychological Bulletin, 147(10), 987-1011. https://doi.org/10.1037/bul0000343

Hughes, J. (2017). paramtest: Run a Function Iteratively While Varying Parameters. https://CRAN.R-project.org/package=paramtest

Hyde, J. S. (1990). Meta-Analysis and the Psychology of Gender Differences. Signs: Journal of Women in Culture and Societ, 16(1), 55-73.

Hyde, J. S. (2005). The gender similarities hypothesis. American Psychologist, 60(6), 581-592. https://doi.org/10.1037/0003-066X.60.6.581

Hyde, J. S. (2014). Gender Similarities and Differences. Annual Review of Psychology, 65(1), 373-398. https://doi.org/10.1146/annurev-psych-010213-115057

Jamnik, M. R., \& Lane, D. J. (2017). The Use of Reddit as an Inexpensive Source for HighQuality Data. https://doi.org/10.7275/J18T-C009

John, O. P., Naumann, L. P., \& Soto, C. J. (2008). Paradigm shift to the integrative Big Five trait taxonomy: History, measurement, and conceptual issues. In Handbook of personality: Theory and research, 3rd ed. (pp. 114-158). The Guilford Press. 
Jones, J. M. (2021, February 24). LGBT Identification Rises to 5.6\% in Latest U.S. Estimate. https://news.gallup.com/poll/329708/lgbt-identification-rises-latest-estimate.aspx

Jorgensen, T. D., Pornprasertmanit, S., Schoemann, A. M., \& Rosseel, Y. (2021). VextttsemTools: Useful tools for structural equation modeling. https://CRAN.Rproject.org/package $=$ sem Tools

Jorm, A. F., Korten, A. E., Rodgers, B., Jacomb, P. A., \& Christensen, H. (2002). Sexual orientation and mental health: Results from a community survey of young and middle aged adults. British Journal of Psychiatry, 180(5), 423-427. https://doi.org/10.1192/bjp.180.5.423

Junkins, E. J., Dugan, K. A., \& Derringer, J. (in prep). Exclusion of LGBTQ+ and TGNC Folks in Romantic Relationships Research. https://doi.org/10.17605/OSF.IO/VKJSY

Junkins, E. J., Dugan, K., Chehab, S., Han, Y. S., Liu, T., Yuan, J., \& Derringer, J. (2022). Systematic Review of the Exclusion of LGBTQIA+ and Nonbinary People in Romantic Relationships Research [Preprint]. PsyArXiv. https://doi.org/10.31234/osf.io/8c9ju

Junkins, E. J., Potter, J. E., Rentfrow, P. J., Gosling, S. D., Potter, J., Harden, K. P., TuckerDrob, E. M., Derringer, J., \& Briley, D. A. (2021). Geographic variation in personality is associated with fertility across the United States. Personality Science, 2, e7275. https://doi.org/10.5964/ps.7275

Kassambara, A. (2021). rstatix: Pipe-Friendly Framework for Basic Statistical Tests. https://CRAN.R-project.org/package=rstatix

Kinsey, A. C., Pomeroy, W. R., \& Martin, C. E. (2003). Sexual Behavior in the Human Male. American Journal of Public Health, 93(6), 894-898. https://doi.org/10.2105/AJPH.93.6.894 
Kling, K. C., Hyde, J. S., Showers, C. J., \& Buswell, B. N. (1999). Gender Differences in SelfEsteem: A Meta-Analysis. 31.

Lewis, N. A. (2021). What counts as good science? How the battle for methodological legitimacy affects public psychology. American Psychologist, 76(8), 1323-1333. https://doi.org/10.1037/amp0000870

Lippa, R. A. (2009). Sex Differences in Sex Drive, Sociosexuality, and Height across 53 Nations: Testing Evolutionary and Social Structural Theories. Archives of Sexual Behavior, 38(5), 631-651. https://doi.org/10.1007/s10508-007-9242-8

Lippa, R. A. (2020). Interest, Personality, and Sexual Traits That Distinguish Heterosexual, Bisexual, and Homosexual Individuals: Are There Two Dimensions That Underlie Variations in Sexual Orientation? Archives of Sexual Behavior, 49(2), 607-622. https://doi.org/10.1007/s10508-020-01643-9

Luong, R., \& Lomanowska, A. M. (2021). Evaluating Reddit as a Crowdsourcing Platform for Psychology Research Projects. Teaching of Psychology, 009862832110207. https://doi.org/10.1177/00986283211020739

Maes, M., Qualter, P., Vanhalst, J., Van den Noortgate, W., \& Goossens, L. (2019). Gender Differences in Loneliness across the Lifespan: A Meta-Analysis. European Journal of Personality, 33(6), 642-654. https://doi.org/10.1002/per.2220

Magliozzi, D., Saperstein, A., \& Westbrook, L. (2016). Scaling Up: Representing Gender Diversity in Survey Research. Socius: Sociological Research for a Dynamic World, 2, 237802311666435. https://doi.org/10.1177/2378023116664352

McEwan, B. (2020). Sampling and validity. Annals of the International Communication Association, 44(3), 235-247. https://doi.org/10.1080/23808985.2020.1792793 
Meehl, P. E. (1992). Factors and Taxa, Traits and Types, Differences of Degree and Differences in Kind. Journal of Personality, 60(1), 117-174. https://doi.org/10.1111/j.14676494.1992.tb00269.x

Meyer, I. H. (2003). Prejudice, social stress, and mental health in lesbian, gay, and bisexual populations: Conceptual issues and research evidence. Psychological Bulletin, 129(5), 674-697. https://doi.org/10.1037/0033-2909.129.5.674

Meyer, I. H. (2007). Prejudice, Social Stress, and Mental Health in Lesbian, Gay, and Bisexual Populations: Conceptual Issues and Research Evidence. 39.

Miguel, Y. M.-S., \& Tobias, S. (Eds.). (2019). 11. LGB within the T: Sexual Orientation in the National Transgender Discrimination Survey and Implications for Public Policy. In Y. M.-S. Miguel, S. Tobias, G. Beemyn, S. R. Rankin, P. Park, L. Crawford, V. Keja, J. Chen, T. Beauchamp, N. B. Burke, A. Z. Aizura, M. C. Enriquez, A. R. de Ruiz, M. Ochoa, J. L. Herman, S. J. Hwahng, \& A. F. Enke, Trans Studies (pp. 172-188). Rutgers University Press. https://doi.org/10.36019/9780813576435-013

Mook, D. G. (1983). In defense of external invalidity. American Psychologist, 38(4), 379-387. https://doi.org/10.1037/0003-066X.38.4.379

Morales, N. A. (n.d.). GENDER AND THE HEXACO MODEL OF PERSONALITY: METAANALYSIS, MEASUREMENT EQUIVALENCE, AND SPECIFICATION OF AGENCY AS A CORE CONSTRUCT OF INVESTMENT. 58.

Mõttus, R., Wood, D., Condon, D. M., Back, M. D., Baumert, A., Costantini, G., Epskamp, S., Greiff, S., Johnson, W., Lukaszewski, A., Murray, A., Revelle, W., Wright, A. G. C., Yarkoni, T., Ziegler, M., \& Zimmermann, J. (2020). Descriptive, Predictive and 
Explanatory Personality Research: Different Goals, Different Approaches, but a Shared Need to Move Beyond the Big Few Traits. 27.

Mustanski, B. S., Garofalo, R., \& Emerson, E. M. (2010). Mental Health Disorders, Psychological Distress, and Suicidality in a Diverse Sample of Lesbian, Gay, Bisexual, and Transgender Youths. American Journal of Public Health, 100(12), 2426-2432. https://doi.org/10.2105/AJPH.2009.178319

Nagata, J. M., Ganson, K. T., \& Austin, S. B. (2020). Emerging trends in eating disorders among sexual and gender minorities. Current Opinion in Psychiatry, 33(6), 562-567. https://doi.org/10.1097/YCO.0000000000000645

National Institutes of Health. (2021). NIH strategic plan to advance research on the health and well-being of sexual \& gender minorities (SGMs) for FY 2021-2025. National Institutes of Health Sexual and Gender Minority Research Office. https://dpcpsi.nih.gov/sites/default/files/SGMStrategicPlan_2021_2025.pdf

Olson, K. R., Key, A. C., \& Eaton, N. R. (2015). Gender Cognition in Transgender Children. Psychological Science, 26(4), 467-474. https://doi.org/10.1177/0956797614568156

Pérez-Stable, E. J. (2016). Sexual and Gender Minorities Formally Designated as a Health Disparity Population for Research Purposes (Director's Message). National Institutes of Health. https://www.nimhd.nih.gov/about/directors-corner/messages/message_10-0616.html

Peterson, R. A., \& Merunka, D. R. (2014). Convenience samples of college students and research reproducibility. Journal of Business Research, 67(5), 1035-1041. https://doi.org/10.1016/j.jbusres.2013.08.010 
Quinn, G. P., Pratt-Chapman, M. L., Meersman, S. C., Chang, S., Kamen, C. S., Maingi, S., Merrill, J. K., \& Schabath, M. B. (2021). Barriers and facilitators to sexual orientation and gender identity (SOGI) data collection. Journal of Clinical Oncology, 39(15_suppl), e18520-e18520. https://doi.org/10.1200/JCO.2021.39.15_suppl.e18520

R Core Team. (2019). R: A Language and Environment for Statistical Computing. R Foundation for Statistical Computing. https://www.R-project.org/

Reis, H. T., \& Carothers, B. J. (2014). Black and White or Shades of Gray: Are Gender Differences Categorical or Dimensional? Current Directions in Psychological Science, 23(1), 19-26. https://doi.org/10.1177/0963721413504105

Ridner, S. L., Topp, R., \& Frost, K. L. (2007). Methodological Issues in Identifying Sexuality for Research. American Journal of Men's Health, 1(1), 87-90. https://doi.org/10.1177/1557988306294609

Riva, G., Teruzzi, T., \& Anolli, L. (2003). The Use of the Internet in Psychological Research: Comparison of Online and Offline Questionnaires. CyberPsychology \& Behavior, 6(1), 73-80. https://doi.org/10.1089/109493103321167983

Rodriguez-Seijas, C., Fields, E. C., Bottary, R., Kark, S. M., Goldstein, M. R., Kensinger, E. A., Payne, J. D., \& Cunningham, T. J. (2020). Comparing the Impact of COVID-19-Related Social Distancing on Mood and Psychiatric Indicators in Sexual and Gender Minority (SGM) and Non-SGM Individuals. Frontiers in Psychiatry, 11, 590318. https://doi.org/10.3389/fpsyt.2020.590318

Ross, L. E., Salway, T., Tarasoff, L. A., MacKay, J. M., Hawkins, B. W., \& Fehr, C. P. (2018). Prevalence of Depression and Anxiety Among Bisexual People Compared to Gay, Lesbian, and Heterosexual Individuals:A Systematic Review and Meta-Analysis. The 
Journal of Sex Research, 55(4-5), 435-456.

https://doi.org/10.1080/00224499.2017.1387755

Rosseel, Y. (2012). lavaan: An R Package for Structural Equation Modeling. Journal of Statistical Software, 48(2), 1-36.

Schmitt, D. P., Long, A. E., McPhearson, A., O’Brien, K., Remmert, B., \& Shah, S. H. (2017). Personality and gender differences in global perspective: GENDER AND PERSONALITY. International Journal of Psychology, 52, 45-56. https://doi.org/10.1002/ijop.12265

Schmitt, D. P., Realo, A., Voracek, M., \& Allik, J. (2008). Why can't a man be more like a woman? Sex differences in Big Five personality traits across 55 cultures. Journal of Personality and Social Psychology, 94(1), 168-182. https://doi.org/10.1037/00223514.94.1.168

Schönbrodt, F. D., \& Perugini, M. (2013). At what sample size do correlations stabilize? Journal of Research in Personality, 47(5), 609-612. https://doi.org/10.1016/j.jrp.2013.05.009

Shatz, I. (2017). Fast, Free, and Targeted: Reddit as a Source for Recruiting Participants Online. Social Science Computer Review, 35(4), 537-549. https://doi.org/10.1177/0894439316650163

Soto, C. J. (2019). How Replicable Are Links Between Personality Traits and Consequential Life Outcomes? The Life Outcomes of Personality Replication Project. Psychological Science, 30(5), 711-727. https://doi.org/10.1177/0956797619831612

Soto, C. J., \& John, O. P. (2017). The next Big Five Inventory (BFI-2): Developing and assessing a hierarchical model with 15 facets to enhance bandwidth, fidelity, and 
predictive power. Journal of Personality and Social Psychology, 113(1), 117-143. https://doi.org/10.1037/pspp0000096

Subbiah, M., \& Rajeswaran, V. (2017). proportion: A comprehensive R package for inference on single Binomial proportion and Bayesian computations. SoftwareX, 6, 36-41. https://doi.org/10.1016/j.softx.2017.01.001

Svetina, D., Rutkowski, L., \& Rutkowski, D. (2020). Multiple-Group Invariance with Categorical Outcomes Using Updated Guidelines: An Illustration Using M plus and the lavaan/semTools Packages. Structural Equation Modeling: A Multidisciplinary Journal, 27(1), 111-130. https://doi.org/10.1080/10705511.2019.1602776

Tierney, N., Cook, D., McBain, M., \& Fay, C. (2021). naniar: Data Structures, Summaries, and Visualisations for Missing Data. https://CRAN.R-project.org/package=naniar

Walton, M. T., Lykins, A. D., \& Bhullar, N. (2016). Beyond Heterosexual, Bisexual, and Homosexual: A Diversity in Sexual Identity Expression. Archives of Sexual Behavior, 45(7), 1591-1597. https://doi.org/10.1007/s10508-016-0778-3

Wilson, B. D. M., \& Meyer, I. H. (2021). Nonbinary LGBTQ Adults in the United States. The Williams Institute. https://williamsinstitute.law.ucla.edu/publications/nonbinary-lgbtqadults-us/

Wu, H., \& Estabrook, R. (2016). Identification of Confirmatory Factor Analysis Models of Different Levels of Invariance for Ordered Categorical Outcomes. Psychometrika, 81(4), 1014-1045. https://doi.org/10.1007/s11336-016-9506-0

Zell, E., Krizan, Z., \& Teeter, S. R. (2015). Evaluating gender similarities and differences using metasynthesis. American Psychologist, 70(1), 10-20. https://doi.org/10.1037/a0038208 
Zietsch, B. P., \& Sidari, M. J. (2020). The Kinsey scale is ill-suited to most sexuality research because it does not measure a single construct. Proceedings of the National Academy of Sciences, 117(44), 27080-27080. https://doi.org/10.1073/pnas.2015820117

Zoeterman, S. E., \& Wright, A. J. (2014). The Role of Openness to Experience and Sexual Identity Formation in LGB Individuals: Implications for Mental Health. Journal of Homosexuality, 61(2), 334-353. https://doi.org/10.1080/00918369.2013.839919

\section{Contributions}

Contributed to conception and design: EJJ, KP, JD

Contributed to acquisition of data: EJJ

Contributed to analysis and interpretation of data: EJJ

Drafted and/or revised the article: EJJ, JD

Approved the submitted version for publication: EJJ, KP, JD

\section{Funding}

There is no funding to report.

\section{Competing interests}

The authors have no competing interests to disclose.

\section{Data accessibility}


The study materials and analysis scripts used for this article can be accessed at https://osf.io/kcqv8/?view_only=55bfc45eeeb144e696501c07c789dd38. The raw data is not available publicly, only scale measures are openly available at the same repository. Open text responses and variables created from these text responses are not available, unless upon reasonable request. 